\title{
Histopathologic subtype-specific genomic profiles of renal cell carcinomas identified by high-resolution whole-genome single nucleotide polymorphism array analysis
}

\author{
AKIRA YOKOMIZO $^{1}$, KEN YAMAMOTO ${ }^{2}$, KENJI FURUNO ${ }^{2}$, MASAKI SHIOTA ${ }^{1}$, \\ KATSUNORI TATSUGAMI $^{1}$, KENTARO KUROIWA ${ }^{1}$ and SEIJI NAITO ${ }^{1}$ \\ ${ }^{1}$ Department of Urology, Graduate School of Medical Science; \\ ${ }^{2}$ Division of Molecular Population Genetics, Department of Molecular Genetics, \\ Medical Institute of Bioregulation, Kyushu University, Fukuoka 812-8582, Japan
}

Received July 7, 2010; Accepted September 9, 2010

DOI: $10.3892 / \mathrm{ol} .2010 .187$

\begin{abstract}
To elucidate the novel and common genetic changes in histopathologic subtype-specific profiles of renal cell carcinomas (RCCs), a newly developed high-resolution wholegenome array analysis was applied. Human CNV370-Duo DNA Analysis BeadChip (genotype 370K) was used in a panel of 22 primary clear cell RCCs (ccRCCs), seven papillary RCCs (PRCCs) (six type II and one type I) and eight chromophobe RCCs (ChRCCs). In ccRCC, a chromosome 3p loss was identified in $95 \%$ of the carcinomas, suggesting that $3 p$ loss is the first stage in ccRCC carcinogenesis. Other frequent changes included losses of $1 p(23 \%), 3 q(46 \%)$ and $8 p(32 \%)$, and gains of $5 q(32 \%)$, $7 \mathrm{p}(27 \%), 7 \mathrm{q}(27 \%)$ and 1q (23\%). The most frequent chromosomal losses in PRCC (43\%) were noted in 3p and 3q, followed by $29 \%$ of losses of 1p, 1q, 11q, 18q, 22p and 22q, and gains of 20q (57\%), 20p (43\%), 8q (43\%) and 12q (43\%). Loss of the entire chromosomes $1,2,6,8,10,13$ or 17 was noted in patients with ChRCC. A high-density single nucleotide polymorphism array analysis confirmed that partial chromosomal changes rarely occur in ChRCC. Additionally, 32 microdeletions and 10 microamplifications of less than $1 \mathrm{Mb}$ were detected, which may represent potential candidate tumor suppressor genes and oncogenes, respectively.
\end{abstract}

\section{Introduction}

Renal cell carcinomas (RCCs) constitute $2-3 \%$ of adult cancers and include clear cell RCC (ccRCC) (75\%), papillary RCC (PRCC) (10\%) and chromophobe RCC (ChRCC) (5\%)

\footnotetext{
Correspondence to: Dr Akira Yokomizo, Department of Urology, Graduate School of Medical Science, Kyushu University, Fukuoka 812-8582, Japan

E-mail: yokoa@uro.med.kyushu-u.ac.jp
}

Key words: single nucleotide polymorphism array, comparative genomic hybridization, renal cell carcinoma subtypes $(1,2)$. Accurate classification of RCCs is crucial, since different histopathologic subtypes require specific therapeutic management strategies due to their markedly different prognoses and responses to therapy (3). The von HippelLindau (VHL) tumor suppressor gene at chromosome 3p25 is inactivated in over $70 \%$ of sporadic ccRCCs, and constitutional VHL mutation carriers have a high lifetime risk of developing ccRCC (4). Molecular-targeting drugs for advanced RCC were designed based on the VHL-hypoxia inducible factor (HIF), vascular endothelial growth factor receptor (VEGFR), plateletderived growth factor (PDGF) and transforming growth factor transduction pathways (5), as well as the mammalian target of the rapamycin pathway (6). However, the therapeutic effect of molecular-targeting drugs in PRCC and ChRCC is less clear, since fewer studies are available on the genetic alterations in these subtypes than those in ccRCC. Therefore, newly developed high-density single nucleotide polymorphism (SNP) array was applied to investigate novel and common genetic change in the three RCC subtypes.

A number of cytogenetic studies have identified the chromosomal region responsible for RCC. Recent advances in array-based comparative genomic hybridization (CGH) technology have allowed chromosomal regions to be examined in much more detail, thus revolutionizing understanding of gene-copy abnormalities (7). The introduction of high-density SNP genotyping technology to genomic profiling, termed SNP-CGH, is a further advance, since the simultaneous measurement of signal intensity variations and changes in allelic composition allows for the detection of copy number changes and copy-neutral loss-of-heterozygosity (LOH) events (8). Furthermore, SNP-CGH has the advantage that candidate genes are readily accessed via the SNP tag number. A few SNP-CGH studies have been performed in RCC, using the $10 \mathrm{~K}$ array (2) and the $307 \mathrm{~K}$ array in ccRCC (9). In this study, SNP-CGH was used to detect chromosomal aberrations in each of the three types of RCC using whole-genome genotyping with Human CNV370-Duo DNA Analysis BeadChip, which covers the $370 \mathrm{~K}$ SNPs over a median spacing of $4.9 \mathrm{~kb}$ in the human genome. 


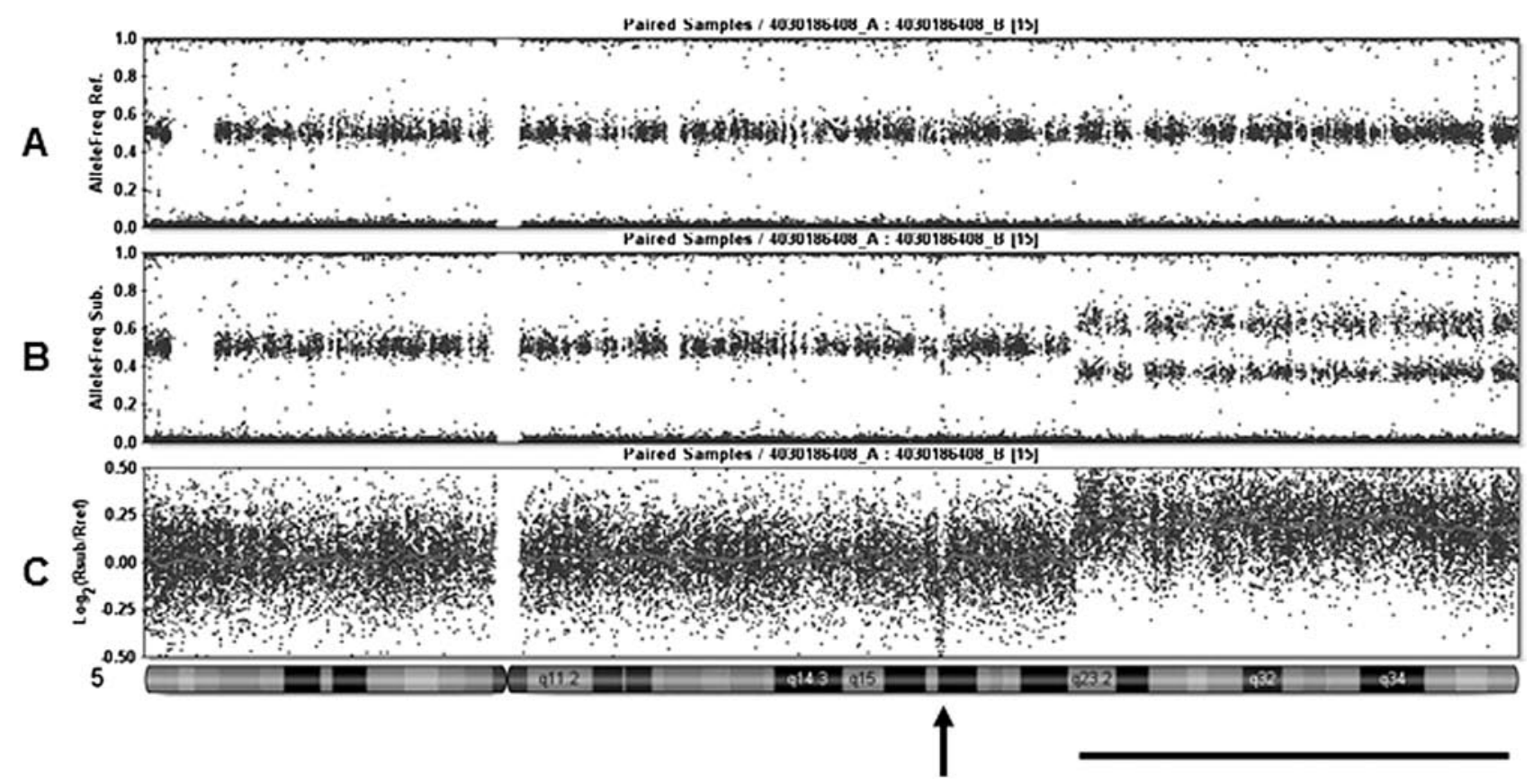

Figure 1. Representative result using the Human CNV-Duo DNA Analysis BeadChip system. SNP typing of (A) normal DNA, (B) tumor DNA and (C) $\log _{2}$ ratio (tumor/normal) is shown. A focal deletion $(\uparrow)$ and partial chromosomal amplification of $5 \mathrm{q}$ (underlined section) are indicated.

\section{Materials and methods}

Tumor samples, normal control and DNA extraction. Primary RCC tumors were surgically resected at Kyushu University Hospital. Samples of carcinoma tissue were frozen in liquid nitrogen immediately after surgery and stored in deep freeze at $-80^{\circ} \mathrm{C}$ until use. The RCC specimens were histopathologically diagnosed as pure ccRCC, PRCC or ChRCC by a single pathologist. Mixed histopathologic types of RCC were excluded from this study. The proportion of tumor cells in a tissue section was confirmed to be $>70 \%$ in all of the tumor tissues. The frozen blocks were then subjected to DNA extraction. DNA from peripheral blood was used as the normal control DNA for each patient. DNA extraction was performed according to standard protocols (10). Written informed consent was obtained from the patients. The study was approved by the Institutional Review Board.

Whole-genome SNP array analysis. The Human CNV370-Duo DNA Analysis BeadChip system (Illumina, San Diego, CA, USA) was used, as previously described (11). The allele balance and $\log _{2}$ ratio (tumor/normal) were visualized using the Genome Browser on BeadStudio ver3 (Illumina).

Statistical analysis. Two-tailed Student's t-tests were used to evaluate the allelic changes and clinical factors, such as stage, tumor grade and prognosis (data not shown).

\section{Results}

Fig. 1 shows a representative result using the Human CNV370-Duo DNA Analysis BeadChip system and the allele frequency of each SNP typing from (A) normal DNA, (B) tumor DNA and (C) $\log _{2}$ ratio (tumor/normal). A comparison of the three lines showed that a focal deletion and partial chromosomal amplification of $5 q$ were identical. Since the chromosomal position of analyzed SNP is available on the public database (http://www.ncbi.nlm.nih.gov/SNP/), genes involved in observed chromosomal aberrations are precisely identified. For example, the $1.45 \mathrm{Mb}$ focal deletion shown in Fig. 1 is flanked by rs104114206 and rs105568833. The deletion contains only one protein-coding gene as a candidate tumor suppressor.

The most common genetic loss in ccRCC was identified in 3p, which was found in $95 \%$ (21/22 samples) of the carcinomas (Table I), suggesting that $3 p$ loss is early stage in clear cell carcinogenesis. Other frequent changes were losses of $1 \mathrm{p}(23 \%), 3 \mathrm{q}(46 \%), 8 \mathrm{p}(32 \%), 8 \mathrm{q}(22 \%), 9 \mathrm{p}(27 \%), 9 \mathrm{q}(27 \%)$ and 18q (23\%), and gains of 5q (32\%), 7p (27\%), 7q (27\%) and 1q (23\%) (Table I). Furthermore, microamplifications and microdeletions of $<1 \mathrm{Mb}$ were detected in 4 and 18 regions, respectively, in ccRCC (Table II). Among these, the microdeletion of $10 \mathrm{q} 23.31$ was identified in two tumors. Moreover, five genes, including PTEN, were identified as candidate tumor suppressor genes in the SNP-based genomic database. The other microamplifications and microdeletions observed are shown in Table II.

The most frequent chromosomal losses (43\%) in PRCC were observed in $3 p$ and $3 q$, followed by $29 \%$ of losses in $1 p$, 1q, 11q, 18q, 22p and 22q (Table I). The highest frequencies of chromosomal gains in PRCC were noted in 20q (57\%), 20p (43\%), 8q (43\%) and 12q (43\%). Microamplifications and microdeletions of $<1 \mathrm{Mb}$ were detected in six and nine regions, respectively, in PRCC (Table II). A total of four microdeletions in 10q12-22 were identified (Table II), but the lesions did not overlap each other, suggesting that common tumor suppressor genes are not located in this lesion. A database search for SNPs in microdeleted areas of 8q24.23 and 


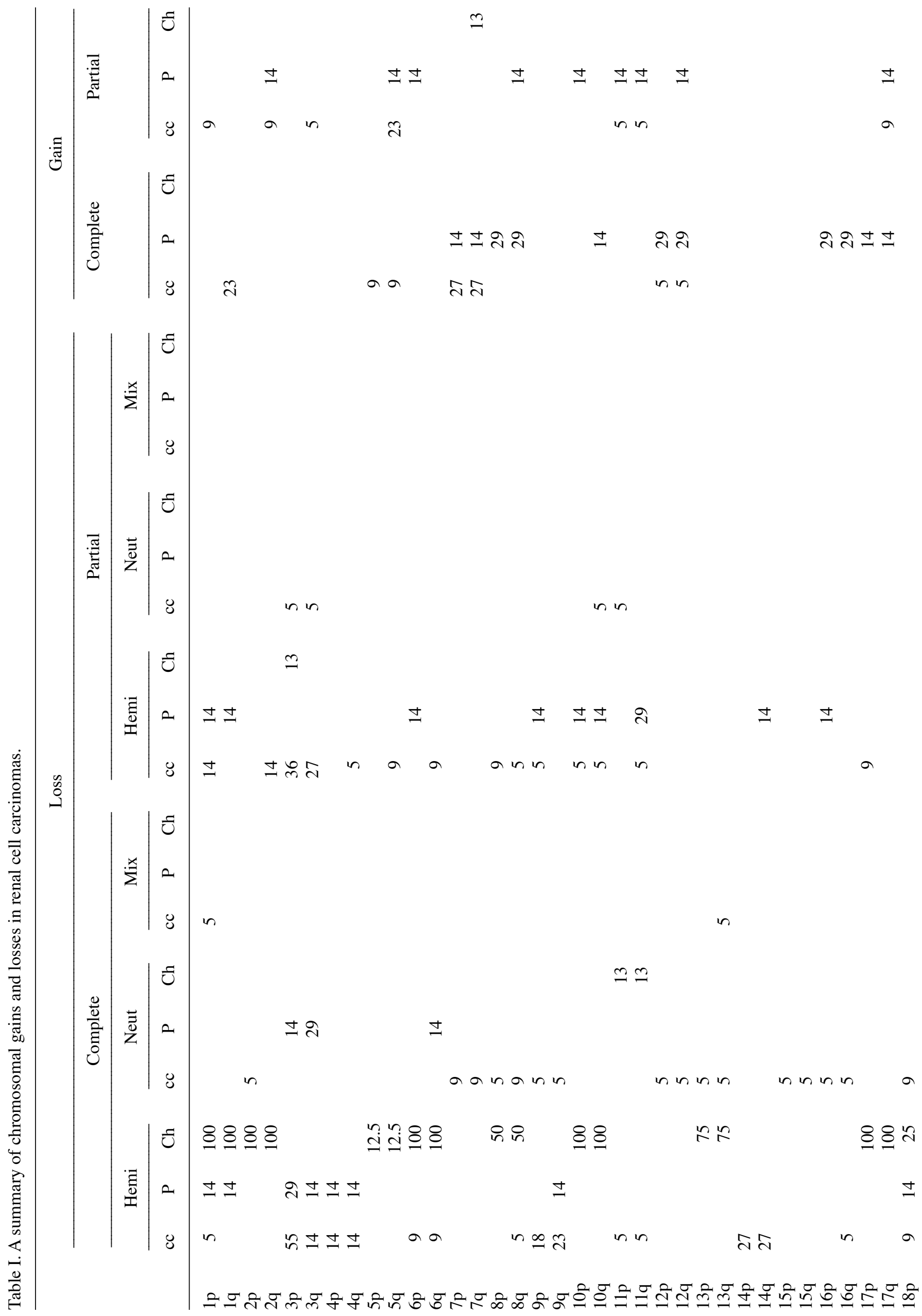




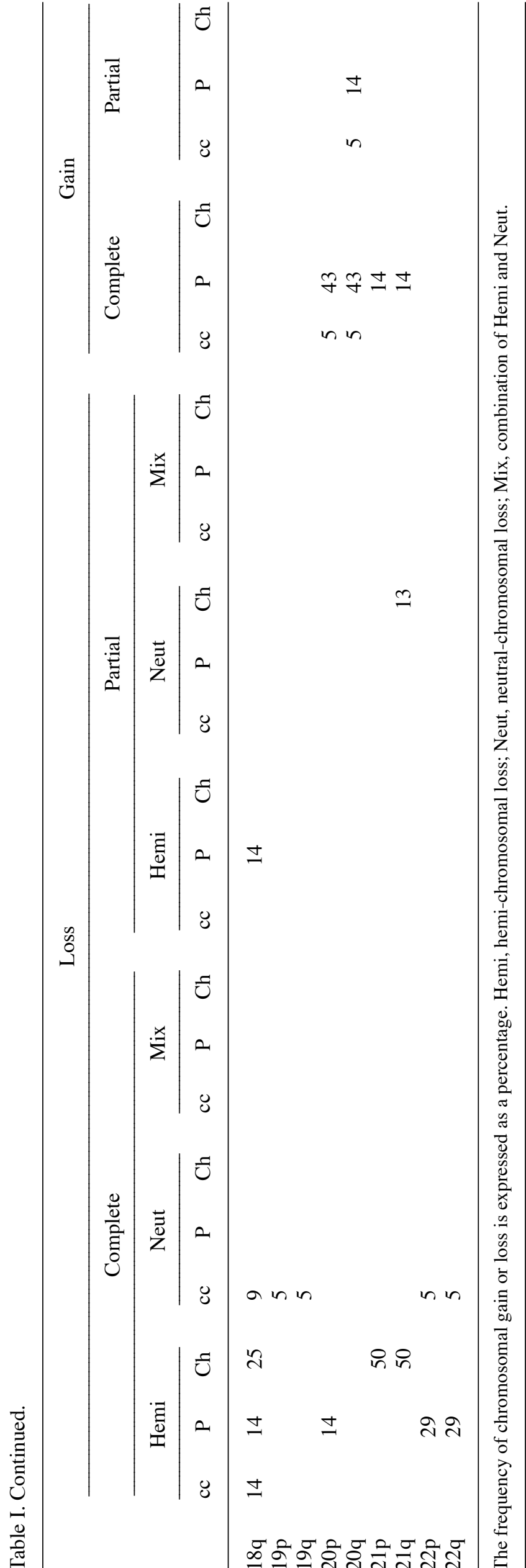

10q21.3 identified only one candidate tumor suppressor gene (data not shown).

By contrast, loss of entire chromosomes was a specific characteristic of the genetic alterations in ChRCC (Tables I and III). The complete allelic loss of the homologous chromosome 1, 2, 6, 10 or 17 was common in the 8 patients with ChRCC (Table III). Furthermore, only one partial chromosomal loss and gain was detected (Table I) and only five microdeletions and no microamplifications were observed in ChRCC (Table II) using high-density SNP array. This result strongly suggests that loss of entire chromosomes is the main event in ChRCC and partial and microgenetic changes rarely contribute to ChRCC carcinogenesis.

Copy-neutral $\mathrm{LOH}$, which was undetectable in previous LOH studies, was identified in a total of 37 chromosomal lesions ( 32 complete and 5 partial losses) (Table I), which is a strong advantage in this SNP array analysis. However, no significant correlations were noted between the allelic changes and clinical factors, such as stage, tumor grade and prognosis (data not shown).

\section{Discussion}

The identification of effective multiple tyrosine kinase inhibitors, including sunitinib and sorafenib, is a crucial development in the treatment of metastatic RCC (5). These drugs inactivate the VHL gene, leading to an accumulation of HIF-1 $\alpha$, followed by the activation of VEGF, PDGF and epidermal growth factors (5). However, the effects of these drugs on PRCC and ChRCC are not well known, since the molecular differences among the different histopathological subtypes of RCC have not been intensively studied and recent trials have mostly been restricted to ccRCC patients (12).

In this study, high-resolution SNP array analysis was used to identify the detailed genomic alterations in the three different histopathologic subtypes of RCC. The results demonstrated that the genetic profiles of the three subtypes were inherently different from each other, while $3 p$ loss was the most frequent change in ccRCC, as well as PRCC. This change suggests that the effectiveness of multiple tyrosine kinase inhibitors should be limited to ccRCC. Notably, the treatment effects of sunitinib and sorafenib in a limited number of patients with PRCC and ChRCC showed poorer clinical responses compared to ccRCC (12). The results of this clinical trial were consistent with the genetic backgrounds identified in the present study.

ccRCCs are characterized by the deletion of the short arm of chromosome $3(4,13)$, loss of $6 \mathrm{p}, 8 \mathrm{p}, 9 \mathrm{pq}$ and $14 \mathrm{q}$ (14). Yoshimoto et al previously analyzed chromosomal copy number aberrations in RCC using array-based CGH, using a genome-wide scanning array with $2304 \mathrm{BAC}$ and PAC clones covering the entire human genome at a resolution of approximately $1.3 \mathrm{Mb}$ (7). In their analysis of $30 \mathrm{ccRCC}$ samples, these authors found losses of 3p25.1-p25.3 (77\%), 3p21.31p22.3 (81\%), 3p14.1-p14.2 (77\%), 8p23.3 (31\%), 9q21.13-qter (19\%) and 14q32.32-qter (38\%), and gains of chromosomes 5q33.1-qter (58\%), 7q11.22-q35 (35\%) and 16p12.3-p13.12 (19\%) (7). Recently, Chen et al (9) reported the SNP profiles of 80 patients with ccRCC determined using Illumina's $307 \mathrm{~K}$ SNP array. These investigators reported that the most common 
Table II. The chromosomal locations of microamplifications (G) and microdeletions (L).

\begin{tabular}{|c|c|}
\hline \multicolumn{2}{|c|}{$\mathrm{ccRCC}$} \\
\hline $\mathrm{G}$ & $1 \mathrm{p} 34.2$ \\
\hline G & $13 q 14.2-14.3$ \\
\hline G & $13 q 22.1-22.2$ \\
\hline G & $5 q 21.2$ \\
\hline $\mathrm{L}$ & $1 \mathrm{p} 36.12-35.3$ \\
\hline $\mathrm{L}$ & $2 q 33.3$ \\
\hline $\mathrm{L}$ & $2 q 14.3$ \\
\hline $\mathrm{L}$ & $2 \mathrm{q} 14.3-2 \mathrm{q} 21.1$ \\
\hline $\mathrm{L}$ & $2 \mathrm{q} 21.2$ \\
\hline $\mathrm{L}$ & $2 \mathrm{q} 32.3$ \\
\hline $\mathrm{L}$ & $4 p 15.33$ \\
\hline $\mathrm{L}$ & $4 p 14$ \\
\hline $\mathrm{L}$ & $5 q 21.3$ \\
\hline $\mathrm{L}$ & $6 q 12$ \\
\hline $\mathrm{L}$ & $6 q 12$ \\
\hline $\mathrm{L}$ & $6 q 12-13$ \\
\hline $\mathrm{L}$ & $6 q 25.1$ \\
\hline $\mathrm{L}$ & $8 \mathrm{q} 21.11$ \\
\hline $\mathrm{L}$ & $10 q 23.31$ \\
\hline $\mathrm{L}$ & $10 q 23.31$ \\
\hline $\mathrm{L}$ & $11 \mathrm{p} 15.1$ \\
\hline $\mathrm{L}$ & $18 \mathrm{q} 12.1$ \\
\hline \multicolumn{2}{|c|}{ PRCC } \\
\hline $\mathrm{G}$ & $2 \mathrm{q} 21.1$ \\
\hline G & 10p12.1 \\
\hline G & $10 \mathrm{p} 12.31$ \\
\hline G & $10 \mathrm{p} 11.22$ \\
\hline G & $10 \mathrm{q} 22.1$ \\
\hline G & $10 \mathrm{q} 22.2$ \\
\hline $\mathrm{L}$ & $1 \mathrm{q} 24.2-25.1$ \\
\hline $\mathrm{L}$ & $8 \mathrm{q} 24.23$ \\
\hline $\mathrm{L}$ & $10 p 15.2-15.1$ \\
\hline $\mathrm{L}$ & $10 \mathrm{q} 21.1$ \\
\hline $\mathrm{L}$ & $10 q 21.3$ \\
\hline $\mathrm{L}$ & $10 q 21.3$ \\
\hline $\mathrm{L}$ & $10 q 22.2$ \\
\hline $\mathrm{L}$ & $14 q 21.2$ \\
\hline $\mathrm{L}$ & $16 \mathrm{p} 13.2$ \\
\hline \multicolumn{2}{|c|}{ ChRCC } \\
\hline $\mathrm{L}$ & $3 \mathrm{p} 22.1$ \\
\hline $\mathrm{L}$ & $14 \mathrm{q} 13.1$ \\
\hline $\mathrm{L}$ & $21 \mathrm{q} 11.2-\mathrm{q} 21.1$ \\
\hline $\mathrm{L}$ & $21 \mathrm{q} 11.12-22.2$ \\
\hline $\mathrm{L}$ & $20 \mathrm{p} 12.1$ \\
\hline
\end{tabular}

ccRCC, clear cell renal cell carcinoma; PRCC, papillary renal cell carcinoma and $\mathrm{ChRCC}$, chromophobe renal cell carcinoma.

LOH was $3 p$ (69 cases), followed by chromosome losses at $8 p$, $6 \mathrm{q}$ and $14 \mathrm{q}$, while the most frequent chromosome gains were at $5 \mathrm{q}$ (32 cases), including 10 entire $5 \mathrm{q}$ amplifications and 21 large amplifications. The results were similar to those of the present study. However, Chen et al only analyzed ccRCCs, and not PRCCs nor ChRCCs.
Table III. The common hemi-chromosomal losses specifically observed in ChRCC.

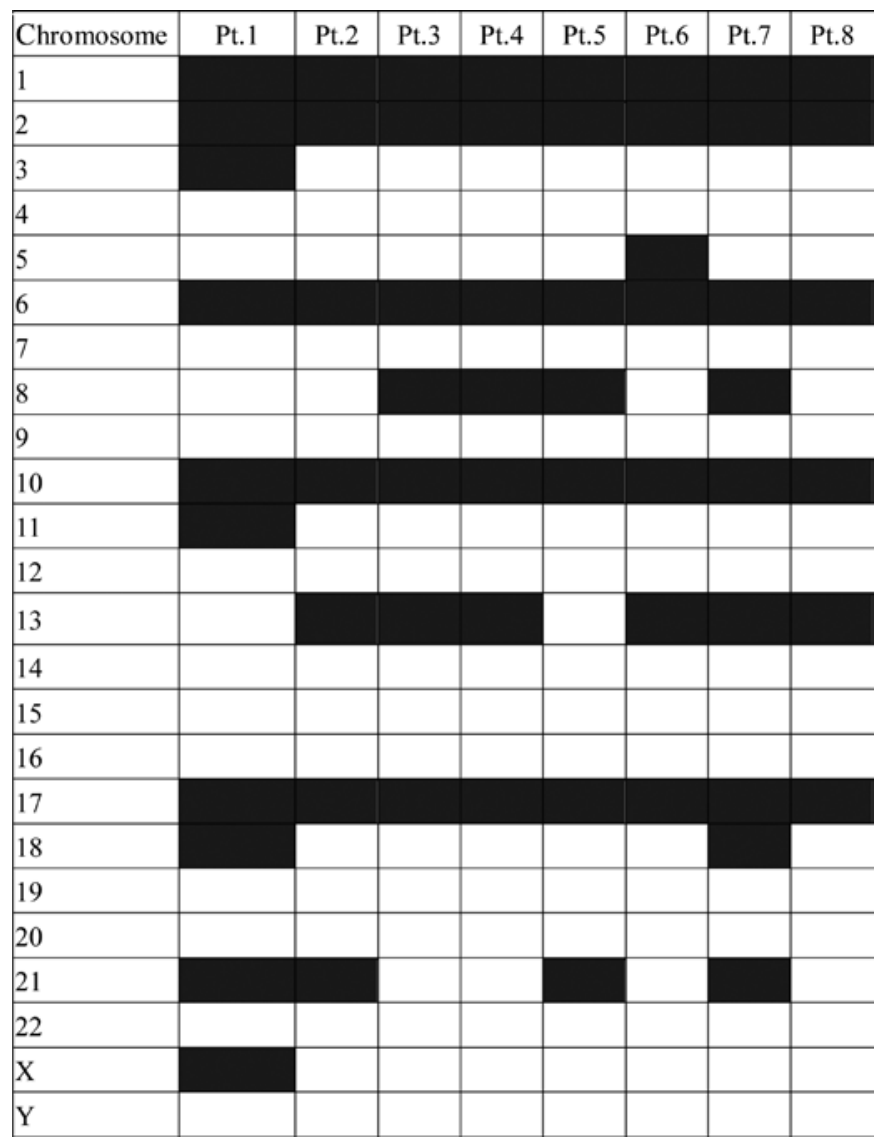

Few studies have used high-density whole-genomic analysis in PRCC. In a study by Klatte et al (15) a cytogenetic analysis was performed to distinguish between the tumor profiles of type I and type II PRCCs. The authors found that loss of chromosome $1 \mathrm{p}$ and $3 \mathrm{p}$, and gain of $5 \mathrm{q}$ were exclusively observed in type II PRCC, whereas trisomy 17 was more frequent in type I (15). Analysis of the genetic profiles of the PRCC cases in the present study, which comprised six cases of type II and one case of type I, were compatible with this previous report, and the single case of type I PCRR showing trisomy 17 (Table I).

We analyzed the patterns of regional gain and loss in the eight samples of ChRCC. The patterns of genomic alterations noted in these ChRCCs differed from those in the ccRCCs and PRCCs. Recurrent genomic losses were detected on chromosomes 1, 2, 6, 8, 10, 13, 17 and 21. Previous studies reported a loss of the entire chromosome arm of chromosomes 1, 2, 6 or 10 ; and $1,2,6,8,10,13,17$ or 21 in ChRCC, using conventional CGH (16) and array-based CGH (7), respectively. Our results confirmed these findings for ChRCC using high-density analysis. We also observed few partial chromosomal changes (Table I) or micro-genetic alterations $(<1 \mathrm{Mb})$ (Table III), suggesting that genetic alterations in small regions, rather than entire chromosomal changes, rarely occur in ChRCC. This entire chromosomal loss may be an initial event in the carcinogenesis of ChRCC.

Contamination with normal cells is occasionally a source of error in the genetic analysis of tumor samples. Peiffer et al 
addressed the effects of tumor heterogeneity and mosaicism on the detection limits and showed that Illumina's SNP array assay was able to detect $\mathrm{LOH}$ in tumor samples combined with $67 \%$ normal stroma (8). We confirmed that the proportion of tumor cells in a tissue section was over $70 \%$, and therefore postulate that this system provided a reliable means of determining the detailed genetic profile of RCCs.

\section{Acknowledgements}

We acknowledge support from the following grants: Health Sciences Research Grants for Clinical Research for Evidenced Based Medicine and Grants-in-Aid for Cancer Research (016), from the Ministry of Health, Labour and Welfare, Japan; Research Promotion Grant from The Tokyo Biochemical Research Foundation, Japan; Grant-in-Aid of Cancer Research from the Fukuoka Cancer Society, Japan. We thank Noriko Hakoda and Seiko Kamori for their technical assistance.

\section{References}

1. Thoenes W, Storkel S and Rumpelt HJ: Histopathology and classification of renal cell tumors (adenomas, oncocytomas and carcinomas). The basic cytological and histopathological elements and their use for diagnostics. Pathol Res Pract 181: 125-143, 1986.

2. Monzon FA, Hagenkord JM, Lyons-Weiler MA, et al: Whole genome SNP arrays as a potential diagnostic tool for the detection of characteristic chromosomal aberrations in renal epithelial tumors. Mod Pathol 21: 599-608, 2008.

3. Herrmann E, Brinkmann OA, Bode ME, et al: Histologic subtype of metastatic renal cell carcinoma predicts response to combined immunochemotherapy with interleukin 2 , interferon alpha and 5-fluorouracil. Eur Urol 51: 1625-1631, 2007.
4. Clifford SC, Prowse AH, Affara NA, Buys CH and Maher ER: Inactivation of the von Hippel-Lindau (VHL) tumour suppressor gene and allelic losses at chromosome arm $3 p$ in primary renal cell carcinoma: evidence for a VHL-independent pathway in clear cell renal tumourigenesis. Genes Chromosomes Cancer 22: 200-209, 1998

5. Eto $\mathrm{M}$ and Naito $\mathrm{S}$ : Molecular targeting therapy for renal cell carcinoma. Int J Clin Oncol 11: 209-213, 2006.

6. Mulders P: Vascular endothelial growth factor and mTOR pathways in renal cell carcinoma: differences and synergies of two targeted mechanisms. BJU Int 104: 1585-1589, 2009.

7. Yoshimoto T, Matsuura K, Karnan S, et al: High-resolution analysis of DNA copy number alterations and gene expression in renal clear cell carcinoma. J Pathol 213: 392-401, 2007.

8. Peiffer DA, Le JM, Steemers FJ, et al: High-resolution genomic profiling of chromosomal aberrations using Infinium wholegenome genotyping. Genome Res 16: 1136-1148, 2006.

9. Chen M, Ye Y, Yang $\mathrm{H}$, et al: Genome-wide profiling of chromosomal alterations in renal cell carcinoma using highdensity single nucleotide polymorphism arrays. Int J Cancer 125: 2342-2348, 2009.

10. Yokomizo A, Mai M, Tindall DJ, et al: Overexpression of the wild type p73 gene in human bladder cancer. Oncogene 18: 1629-1633, 1999

11. Pfeifer D, Pantic M, Skatulla I, et al: Genome-wide analysis of DNA copy number changes and LOH in CLL using high-density SNP arrays. Blood 109: 1202-1210, 2007.

12. Choueiri TK, Plantade A, Elson P, et al: Efficacy of sunitinib and sorafenib in metastatic papillary and chromophobe renal cell carcinoma. J Clin Oncol 26: 127-131, 2008.

13. Higgins JP: Gene array studies in renal neoplasia. ScientificWorldJournal 6: 502-511, 2006.

14. Thrash-Bingham CA, Salazar H, Freed JJ, Greenberg RE and Tartof KD: Genomic alterations and instabilities in renal cell carcinomas and their relationship to tumor pathology. Cancer Res 55: 6189-6195, 1995.

15. Klatte T, Pantuck AJ, Said JW, et al: Cytogenetic and molecular tumor profiling for type 1 and type 2 papillary renal cell carcinoma. Clin Cancer Res 15: 1162-1169, 2009.

16. Speicher MR, Schoell B, du Manoir S, et al: Specific loss of chromosomes $1,2,6,10,13,17$, and 21 in chromophobe renal cell carcinomas revealed by comparative genomic hybridization. Am J Pathol 145: 356-364, 1994. 